\title{
THE ROLE OF RIGHT HEMISPHERE IN RECOVERY OF POST-STROKE APHASIA
}

\author{
Adriana Elena Nica ${ }^{1,2}$, Daniel Alexa ${ }^{3,4}$, Octavian Andronic $^{1}$, Tabita Cazac $^{1}$, \\ Dan Nicolae Paduraru ${ }^{1,2}$ \\ 1“Carol Davila” University of Medicineand Pharmacy, Bucharest \\ ¿University Emergency Hospital, Bucharest \\ ${ }^{3}$ Neurol ogy Clinic, Rehabilitation Hospital, Iasi \\ 4"GrigoreT. Popa" University of Medicineand Farmacy, I asi
}

\begin{abstract}
Previous data obtained especially from rTMS studies indicate that right hemisphere recruitment has a limiting effect on recovery of poststroke aphasia in right handed patients by inhibiting the activation of intact perilesional areas in left hemisphere and preventing them from regaining function. New researches bring new arguments to support the positive influence of right hemisphere on restoration of language and communication. In this paper we try to review the latest evidences regarding the contribution of the right hemisphere to post-stroke aphasia recovery.
\end{abstract}

Keywords: aphasia, right hemisphere, right temporoparietal regions

\section{INTRODUCTION}

Besides motor deficit another redoubtable consequence of stroke affecting the dominant hemisphere is aphasia. Recovering language deficit is a great challenge and its neuronal substrate is still in debate. Classically it is considered that in cases with small lesions language recovery relies on recruiting intact perilesional areas in left hemisphere while in cases with large lesions are activated homotopic areas of the right hemisphere (1). There are some authors who believe that the right hemisphere involvement in the recovery of language plays a harmful, maladaptive role because interfere with recruiting of intact areas from the left hemisphere and prevents them to contribute to language recovery. Some arguments for this theory are provided by the data obtained from studies using inhibitory repetitive transcranial magnetic stimulation (rTMS) which showes improvements of aphasia after blocking the shift of language through corpus callosum towards the right hemisphere. (2) On the other hand there are also opinions that say that the right hemisphere has a beneficial role and one of the strongest arguments is the case of a 72 year old woman who has had aphasia after a left hemisphere stroke with good recovery following a 10 day protocol of inhibitory rTMS upon right hemisphere and who, subsequently, after a second stroke that affected right hemisphere this time, installed an aphasic syndrome more severe than after the first stroke. (3) In this regard, in this review we plan to bring new evidence to clarify the role of the right hemisphere in recovery of aphasia in left hemisphere stroke in right handed patients.

\section{THE ROLE OF RIGHT HEMISPHERE IN APHASIA RECOVERY}

Pani et all used a technique derived from diffusion tensor imaging, fractional anisotropy, to examine the integrity of the arcuate fasciculus in 28 men and 5 women with poststroke aphasia. These patients were undergoing tests to quantify the degree of fluency of language and the results were correlated with values of fractional anisotropy for areas of homologous right hemisphere speech centers, 
the entire corpus callosum, and some selected fiber from corpus callosum. The results were then compared with a healthy control group. It was found that in patients who experienced greater recovery of speech the integrity of white matter from 3 homotops of the right hemisphere connected by arcuate fasciculs was better which shows a degree of involvement of the right hemisphere in the recovery of language. In Pani's interpretation it is not clear whether these anatomical differences are a contributing factor preexisting stroke or are the consequence of poststroke remodeling. (4)

It seems that there are structural differences not only for white matter but also for right hemisphere gray matter between patients with aphasia and those without aphasia after stroke. Xing et all brought new data on the morphology of gray matter in the right hemisphere. They used voxel based morphometry to achieve a lesion-language mapping in three study groups: patients with poststroke aphasia, stroke patients without aphasia and healthy controls. The data obtained show that in patients with aphasia gray matter volume in homologous right hemisphere areas is higher and this hypertrophy contribute at least in part to the recovery of language. Moreover, symptom-lesion mapping showed a differentiation of left regions for different abilities of language. Thus for spontaneous speech, naming, and repetition are involved also the right temporoparietal areas. Cortex volume in these right temporoparietal regions was higher in patients with poststroke aphasia suggesting an independent contribution of dorsal right circuit to the recovery of language, which lead us towards the double current model of language processing proposed by Hickok and Poeppel. (5)

According to this model semantic processing is organized bilaterally. Thus the left hemisphere performs a more accurate coding for syntactic information while semantic representation is better in the right hemisphere. Enabling perilesional and contralateral homologues regions in patients with poststroke aphasia was studied using magnetoencephalography by Kielar et al. They showed that different components of linguistic information are analyzed by specialized networks and compartments of the brain. Inferior frontal areas are bilaterally involved in the selection of semantics and the semantic integration is achieved in temporal regions also bilaterally. Involving areas of the right hemisphere becomes necessary in cases where deciphering the language is more difficult. Studies show that in young people, language processing is easier being executed mainly in the left hemisphere.
Right hemisphere regions could be activated but at a subliminal intensity. Elderly instead activate both hemispheres and in patients with damage of the left hemisphere these areas of the right hemisphere become better trained for language processing and will assume the tasks of left hemisphere. Semantic significance in young and healthy individuals is decrypted in ventral left frontotemporal area but in the elderly appears more involvement of bilateral ventral regions. To organise the syntax in young individuals dorsal frontoparietal regions are activated bilaterally and in the elderly the recruitment is extended more bilaterally. In stroke patients linguistic processing recruite available regions of both hemispheres but unlike in healthy subjects the activation of left ventral frontotemporal regions for semantic anomalies had been reduced and there were involved some intact left temporal and parietal perisylvian areas together with right inferior parietal and posterior temporal cortex. Syntactic anomalies in stroke patients activated parietal regions bilaterally, including the superior parietal cortex and also occipital areas along with superior medial frontal areas and inferior frontal gyrus of the right hemisphere, but this activation was significantly lower in patients compared with controls because they was not able to detect syntactic errors. The results show that in patients with aphasia semantic processing recruited also right hemisphere, namely the right parietal frontotemporal region. Although some frontal cortical areas are recruited too (right dorsolateral frontal gyrus and right inferior frontal gyrus) their contribution is minor and is not reflected in clinical outcomes. Syntactic processing compensation is lower as demonstrated by the lack of response to the syntactic anomalies identified by magnetoencephalography than in healthy controls in whom these syntactic errors determine wider and higher answers comparative with semantic anomalies. The absence of syntactic anomaly detection by aphasic patients show that grammatical processing may be a cognitive characteristic highly vulnerable that can not be easily regained after brain injury. Syntactic processing is generally more susceptible to disruption by brain injuries than semantic processing. Kielar's study shows that recovery of poststroke aphasia involves activating left intact perisylvian areas along with regions in right hemisphere. The results are different for semantic and syntactic processing. Patients with better recovery of semantic activity recruited areas of right ventral temporal and inferior parietal cortex. In contrast, syntactic preservation was mediated by the right and left superior dorsal cortex and right frontal cortex, al- 
though disturbed syntactic processing recover harder after injury of left hemisphere centers. (6)

In line with Kielar's results are those of two studies using data obtained by fMRI.

Sims correlated semantic processing with the size and location of the lesion in 14 patients with chronic poststroke aphasia. The task was to match a written word with a picture or to recognise if two written words are related. It has shown that the best results were obtained when the left inferior frontal gyrus can be used, even if it is affected. If the left hemisphere is severe damaged the right middle temporal gyrus, supramarginal gyrus and angular gyrus are engaged. (7)

More et all investigated brain activation in perilesional areas in the left hemisphere and in right counterparts in 12 patients with chronic aphasia before and after administration of an intensive treatment (3-4 hours of therapy for 10 consecutive days intensive language action therapy). For testing were used 36 sentences with varying degrees of

\section{REFERENCES}

1. D.C. Amitay, Albert M.L. Brain and Language: Evidence for Neural Multifunctionality, Behavioural Neurology, Vol 2014, 9 June, 16 pages

2. Barrett A.M., Hamilton R.H. Drawing on the right brain for aphasia recovery, Neurology ${ }^{\circledR} 2016 ; 86: 1-2$

3. Turkeltaub P.E., Coslett H.B., Thomas H.L., Faseyitan O., Benson J., Norise C., Hamilton R.H. The right hemisphere is not unitary in its role in aphasia recovery, Cortex 48 (2012) 1179 el 186

4. Pani E., Zheng X., Wang J., Norton A., Schlaug G. Right hemisphere structures predict poststroke speech fluency, Neurology 2016;86:1-8

5. Xing S., Lacey E.H., Skipper-Kallal L.M., Jiang X., Harris-Love M.L., Zeng J., Turkeltaub P.E. Right hemisphere grey matter structure and language outcomes in chronic left hemisphere stroke, BRAIN 2016: 139; 227-241 ambiguity. At the end of study all patients had a significant improvement of language due to recruiting of frontal and temporal areas. These areas activation was more intense for sentences with higher ambiguity. (8)

\section{CONCLUSION}

In the light of these recent studies we can emphasize that at least some areas of right hemisphere can be useful in recovering the post stroke aphasia in right handed people. Not only functional data sustain this contribution in language reorganization but also structural changes can be visualized in right white and gray matter. These results have an impact on future approach of aphasia treatment showing that right hemisphere plays an adaptative rol rather than a detrimental one. Inhibitory procedures should be avoided or applied very carefully on specific sites on right hemisphere.

6. Kielar A., Deschamps T., Jokel R., Meltzer J.A. Functional Reorganization of Language Networks for Semantics and Syntax in Chronic Stroke: Evidence From MEG, Human Brain Mapping 00:00-00 (2016)

7. Sims J.A., Kapse K., Glynn P., Sandberg C., Tripodis Y., Kiran S. The relationships between the amount of spared tissue, percent signal change, and accuracy in semantic processing in aphasia, Neuropsychologia 84 (2016)113-126

8. Mohr B., Difrancesco S., Harrington K., Evans S., Pulvermüller F. Changes of right-hemispheric activation after constraint-induced, intensive language action therapy in chronic aphasia: fMRI evidence from auditory semantic processing, Front. Hum. Neurosci., 14 November 2014 | http://dx.doi.org/10.3389/fnhum.2014.00919 